\title{
Orbitoethmoid Metallic Foreign Body
}

\author{
${ }^{1}$ Gauri Belsare, ${ }^{2}$ Archana S Nair, ${ }^{3}$ Snehal Sakhale
}

\begin{abstract}
Intraorbital foreign body extending to sinuses is rare; often such cases are associated with multiple ocular and orbital morbidities. Retained organic foreign bodies can cause severe inflammation and are detected earlier, whereas inorganic foreign bodies are inert and well tolerated except copper. The management of posteriorly located orbital foreign bodies is a therapeutic dilemma. We report an unusual case of posterior orbital foreign body extending to the ethmoids and sphenoid sinus exclusively removed by transnasal endoscopic approach. Transnasal endoscopic approach can be used as an effective and efficient approach for the removal of orbital foreign bodies lodged on the medial side of the orbit. This approach is recommended in all the cases of foreign bodies in the craniofacial region provided that it is approachable through endoscope and expertise and facilities are available.
\end{abstract}

Keywords: Endoscopic sinus surgery, Foreign bodies, Orbitoethmoid.

How to cite this article: Belsare G, Nair AS, Sakhale S. Orbitoethmoid Metallic Foreign Body. Int J Otorhinolaryngol Clin 2016;8(2):84-87.

\section{Source of support: Nil}

Conflict of interest: None

\section{INTRODUCTION}

Foreign bodies in the paranasal sinuses and orbit are rare. It is usually traumatic and rarely iatrogenic. ${ }^{1}$ Foreign body may be organic or inorganic. Retained organic foreign bodies can cause severe inflammation and are detected earlier, whereas inorganic foreign bodies are inert and well tolerated except copper. ${ }^{2}$ The maxillary sinus is the most commonly involved and is often associated with multiple ocular and orbital morbidities, such as conjunctival hemorrhage, optic neuropathy, corneal tear, orbital fracture. ${ }^{3,4}$ High index of suspicion is required to identify foreign body, particularly in the setting of a trivial external injury. We present an unusual case of a large intraorbital foreign body (IoFB) extending to the ethmoid and sphenoid sinuses removed exclusively by transnasal endoscopic approach.

\footnotetext{
${ }^{1}$ Head, ${ }^{2,3}$ Resident

${ }^{1-3}$ Department of ENT, Ruby Hall Clinic, Pune, Maharashtra India

Corresponding Author: Archana S Nair, Resident, Department of ENT, Ruby Hall Clinic, Pune, Maharashtra, India, Phone: +919545550661, e-mail: archanasnair@rediffmail.com
}

\section{CASE REPORT}

Otorhinolaryngology service was called for in the management of a 35-year-old male referred from another hospital following an industrial accident 2 days prior. Patient had sustained superficial burns on the face and neck, had a linear $1 \mathrm{~cm}$ laceration near the lateral canthus below the eyebrow on the left side, and complained of loss of vision in the left eye, pain, and restricted eye movements. His visual acuity was $6 / 6$ on right eye with no perception of light on left. Ocular mobility was restricted in all directions in left eye, and pupil on the left side was dilated. External examination of left eye revealed significant chemosis and conjunctival congestion and proptosis. An X-ray skull and computed tomography (CT) scan were ordered due to the strong suspicion of a foreign body. Computed tomography identified a large metallic foreign body measuring $3 \times 1.5 \mathrm{~cm}$ deep in the posterior orbit near the orbital apex and optic nerve. Part of this foreign body could be seen protruding into sphenoid sinus through lamina papyracea and was only a few millimeters away from the carotid artery (Figs 1 to 3 ).

Even though the patient did not have vision, considering the medial location of the foreign body in the orbit, an endoscopic removal was planned. Transnasal endoscopic approach would be minimally invasive with maximum exposure compared with an external approach in this patient. Surgery was done using 0 and $30^{\circ}$ nasal endoscopes. After uncinectomy, anterior and posterior ethmoidectomy was done and was followed by delineation of the anterior skull base and the lamina papyracea. A metallic

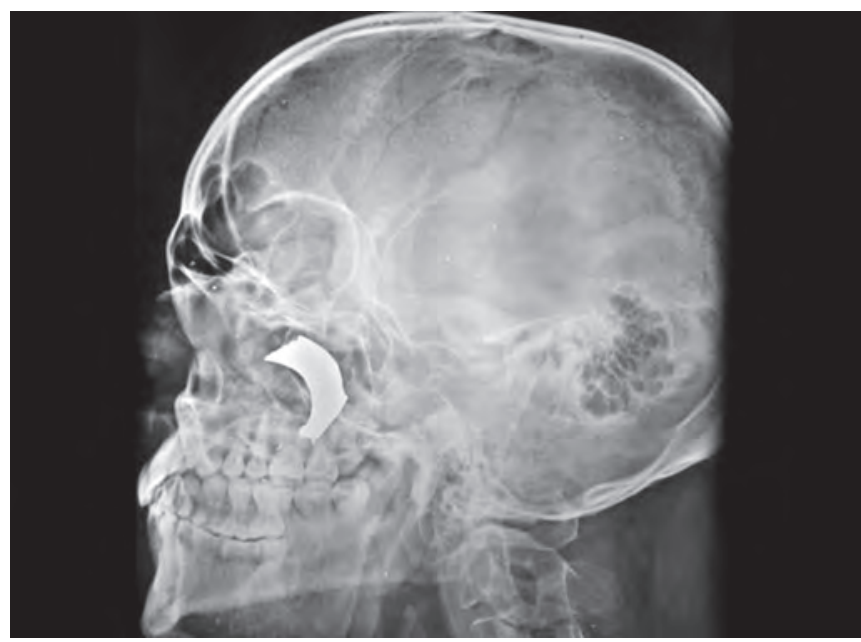

Fig. 1: X-ray showing the location of the foreign body within the orbit extending to the sinuses 


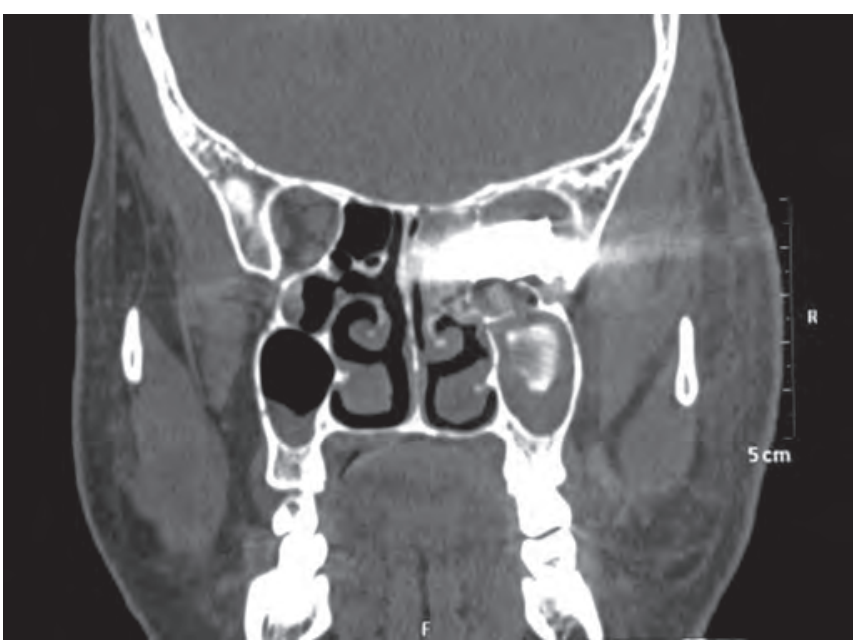

Fig. 2: Computed tomography scan coronal view showing a large metallic foreign body measuring $3 \times 1.5 \mathrm{~cm}$ deep in the posterior orbit near the orbital apex and seen protruding into sphenoid sinus through lamina papyracea, few millimeters away from carotid artery

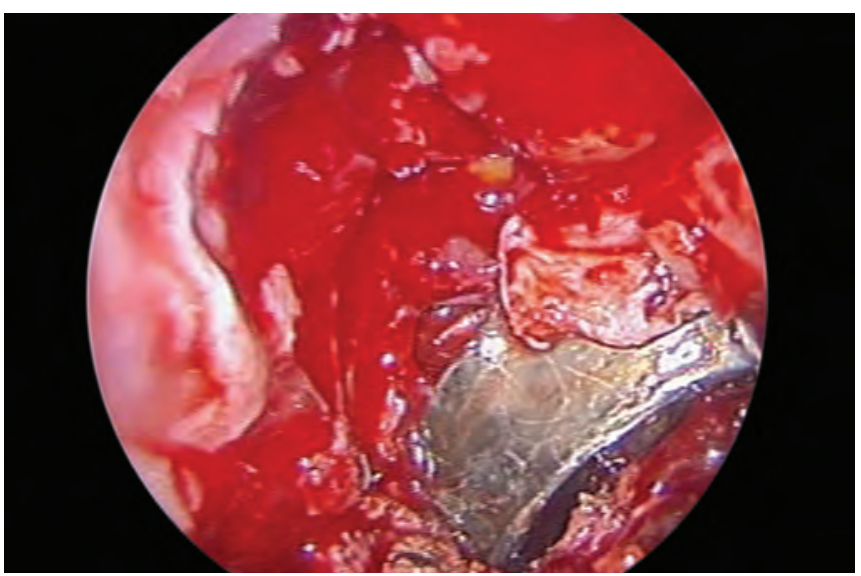

Fig. 4: Intraoperative image of the foreign body seen protruding from the orbit into the ethmoid sinus

disc-shaped body was seen protruding from the orbital apex, disrupting the lamina papyracea and extending to the posterior ethmoid and sphenoid sinus (Fig. 4). With a Freer elevator, it was displaced medially and inferiorly, and after doing a wide sphenoidotomy, it was displaced from the sphenoid and removed with Blakesley forceps (Fig. 5).

The wound was thoroughly cleaned with betadine saline wash. Even though current literature describes the negative impact of betadine on mucociliary clearance of the sinuses, ${ }^{5}$ considering the source of foreign body from a machine part, diluted betadine with lesser ciliotoxic effect ${ }^{6}$ was used for postoperative flushing of sinuses to reduce the risk of postoperative infection. And also the endoscopic surgery was not done in a pathological sinus with preexisting mucociliary clearance impedance. The defect in the lamina papyracea was packed using Gelfoam and Surgicel. Postoperatively, he was started on intravenous antibiotics and steroids to reduce inflammation. On follow-up pain and ocular motility and

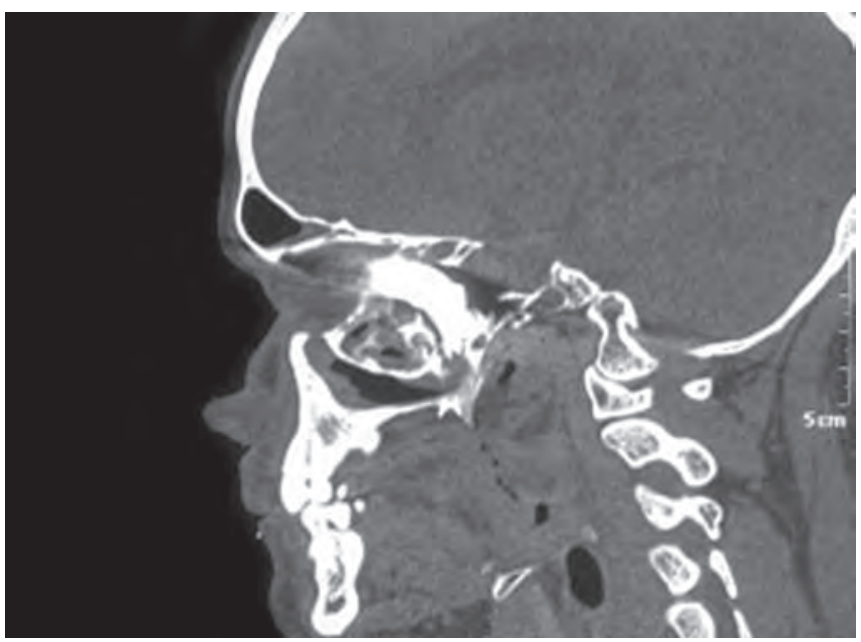

Fig. 3: Computed tomography scan sagittal view showing the large metallic foreign body extending to the ethmoid and sphenoid sinus

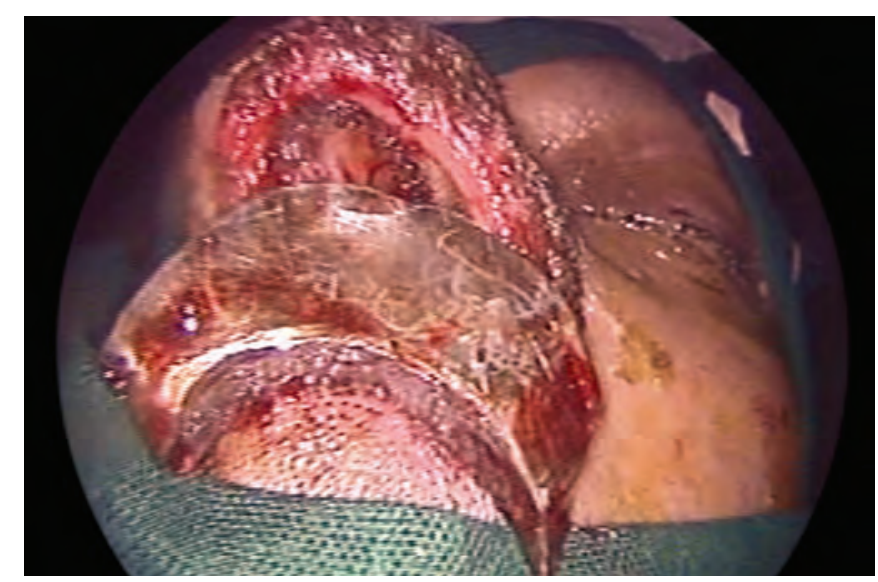

Fig. 5: Foreign body after its removal

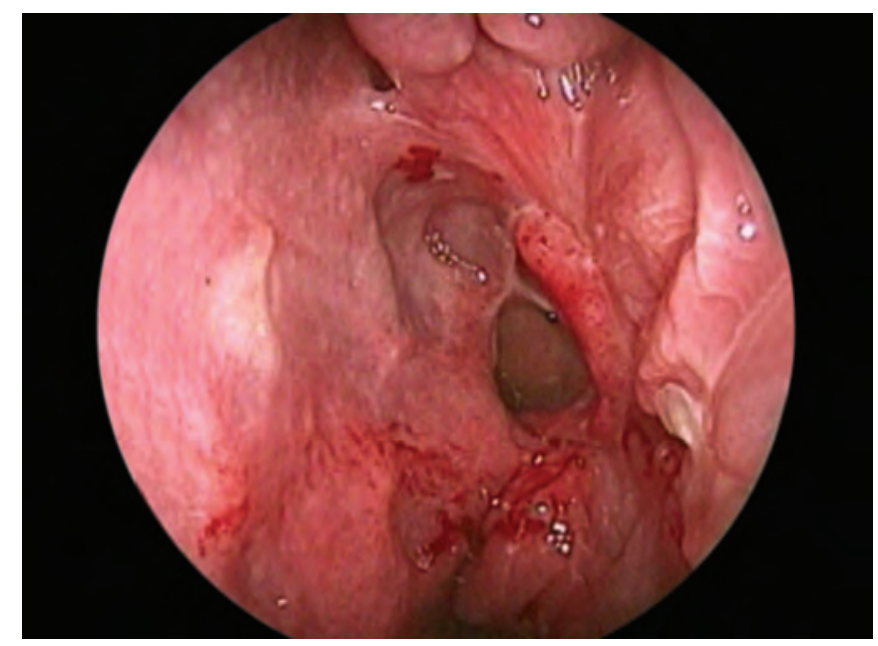

Fig. 6: Postoperative endoscopic image showing the sphenoidotomy and the healthy mucosal lining over the defect on lamina papyracea

proptosis reverted, but there was no improvement in the visual acuity. The defect in lamina papyracea was found satisfyingly healed on follow-up (Fig. 6). 


\section{DISCUSSION}

This case is unusual because of the large size and the intimate location of the foreign body in relation to important structures, such as optic nerve and internal carotid artery, which made the removal of the foreign body more challenging. Considering the scenario that patient already had a nonfunctional eye due to optic nerve transection, which was located in the trajectory of the foreign body, and the medial location of the foreign body in the orbit extending to the sinus, a clinical decision was arrived to remove it via transnasal endoscopic approach. Thereby, morbidity of an extensive external approach could be avoided. We could successfully remove the foreign body endoscopically.

Intraorbital foreign body presents an interesting therapeutic dilemma when located posterior in the orbit. A variety of foreign bodies have been reported in the literature, including glass, stone, metal, wood, graphite, button. The possibility of foreign body should be considered following a history of trauma with persistent signs of inflammation, limited eye movements, and deterioration of vision as in our case. Orbital fat tends to conceal the trajectory, making it difficult to identify a point of entry, and thus, suspicion is crucial for defining the diagnosis. ${ }^{7}$ The cause of limited eye movement should be assessed with care as it may have been caused by the presence of foreign body, trauma to oculomotor, trochlear, and abducens nerve or muscle impingement in fractured orbital bones in orbital walls. ${ }^{8}$ It is first most important to complete a full systemic evaluation and to rule out an ophthalmologic emergency (i.e., ruptured globe). Empiric broad-spectrum antibiotics should be administered to all patients. Appropriate imaging can then be obtained to help identify the foreign body. Computed tomography is preferred as magnetic resonance imaging (MRI) should be avoided until a metallic foreign body can be unequivocally ruled out. Factors, such as foreign body composition, inflammation, infection, functional deficit, and potential for iatrogenic damage are considered when choosing the appropriate treatment. All organic foreign bodies should be removed, although there is a paucity of data on those located in the posterior orbit. The approach to inorganic foreign bodies is more complex. Most retained metallic IoFBs are well tolerated and typically have minimal adverse visual prognosis with the exception of copper and lead. Surgical approach is planned on the basis of the location (anterior or posterior orbit) and the presence of other injuries or foreign body-related complications (such as optic nerve compression, infections, and extraocular muscle involvement). ${ }^{9,10}$
Endoscopic removal can be attempted in medially located foreign bodies. Nasal endoscopes have improved in the last two decades with better rod lenses and fiber optics. These have enabled us to see the intranasal structures better and led to endoscopic-guided surgery, such as functional endoscopic sinus surgery. Once familiar with this approach, surgeons could confidently attempt to carry out other procedures endoscopically, like the removal of foreign bodies. Though not yet a common procedure, endoscopic removal has been documented for foreign bodies of the ethmoid and more frequently of the maxillary sinus. It is safe, simple, and with minimal morbidity. ${ }^{11}$ It gives a distinct advantage of better illumination, magnification, visualization of critical areas, and a scarless surgery. Madina ${ }^{12}$ first reported endoscopic approach to sinus foreign body. In this case, the metallic foreign body was in sphenoethmoidal junction with associated penetrating globe injury and traumatic optic neuropathy. Sino-orbital foreign body can be removed by exclusive endoscope or endoscope assisted along with open exploration..$^{13,14}$ A written consent, in case of need of conventional open approach and slipping of foreign body into airway, needs to be obtained when done under local anesthesia. We had used choanal pack in this patient to prevent slipping of foreign body.

\section{CONCLUSION}

The case demonstrates that transnasal endoscopic approach can be used as an effective and efficient approach for removal of orbital foreign bodies lodged on the medial side of the orbit. This approach is recommended in all the cases of foreign bodies in the craniofacial region provided that it is approachable through endoscope and expertise and facilities are available.

\section{REFERENCES}

1. Donald PJ, Gadre AK. Neuralgia-like symptoms in a patient with an air gun pellet in the ethmoid sinus: a case report. J Laryngol Otol 1995 Jul;109(7):646-649.

2. Nasir AM, Haik BG, Fleming JC, Hailah M, Al-Hussain, Karcioglu ZA. Penetrating orbital injury with organic foreign bodies. J Ophthalmol 1999 Mar;106(3):523-532.

3. Graces SM, Norris CW. Unusual frontal sinus body. J Laryngol Otol 1972 Dec;86(12):1265-1268.

4. Thornwell WC, Williams HL. Foreign body involving the floor of orbit and antrum. Arch Otolaryngol 1944;39(1): 83-85.

5. Kim JH, Rimmer J, Mrad N, Ahmadzada S, Harvey RJ. Betadine has a ciliotoxic effect on ciliated human respiratory cells. J Laryngol Otol 2015 Jan;129 (Suppl 1):45-50.

6. Gosepath J, Grebneva N, Mossikhin S, Mann WJ. Topical antibiotic, antifungal, and antiseptic solutions decrease ciliary activity in nasal respiratory cells. Am J Rhinol 2002 Jan-Feb;16(1):25-31. 
7. Bullock JD, Warwar RE, Bartley GB, Waller RR, Henderson JW. Unusual orbital foreign bodies. Ophthal Plast Reconstr Surg 1999 Jan;15(1):44-51.

8. Macrae JA. Diagnosis and management of a wooden orbital foreign body: case report. Br J Ophthalmol 1979 Dec;63(12): 845-851.

9. Wolfley DE. The lid crease approach to the supromedial orbit. Ophthalmic Surg 1985 Oct;16(10):652-656.

10. Robert JP, Christopher Z, Gary JL Jr. Posterior intraorbital foreign body: take it or leave it? Open Reconstr Cosmet Surg 2008;1:1-3.
11. Davidson TM, Stream G. Extended indication for endoscopic sinus surgery. Ear Nose Throat J 1994 Jul;73(7):467-474.

12. Madina R. Endoscopic sinus surgery: a metallic foreign body at the sphenoethmoidal junction. J Laryngol Otol 1992 Nov;106(11):998-999.

13. Khan-Lim D, Ellis J, Saleh H, Ram B. Endoscopic transnasal removal of orbital foreign body. Eye (Lond) 1999 Oct;13 (Pt 5):667-668.

14. Wu MR, Shih CT, Yeh CW. Transorbital penetrating injury of the paranasal sinuses. J Laryngol Otol 1998 Dec;112(12): 1202-1204. 